\title{
Una revisión sistemática sobre efectividad en psicoterapias breves y focalizadas
}

\section{Resumen:}

El presente estudio de revisión sistemática consiste en relevar publicaciones iberoamericanas de los últimos diez años sobre la efectividad en psicoterapias breves. Se aplicó la declaración PRISMA para revisión sistemática en bases de datos que relevaron artículos científicos en español y portugués los últimos diez años. Se hallaron 38 publicaciones de nueve países Iberoamericanos destacándose las realizadas en España (50\%). Se debate sobre la importancia de realizar investigación sobre los resultados de las políticas de Salud Mental en Iberoamérica. Un crecimiento de estos estudios en la región podría ayudar en una mejora de la administración del recurso sanitario.

\section{Palabras clave:}

Efectividad en psicoterapia- Psicoterapias breves-Atención Primaria de la Salud

\section{Summary:}

This systematic review study survey Ibero-American publications of the last ten years on the effectiveness of short-form psychotherapy. The PRISMA statement was applied for systematic review studies in databases that surveyed Spanish- and Portuguese-language papers in the last ten years. The search resulted in 38 publications from nine Ibero-American countries, the largest portion corresponding to Spain (50\%). The importance of conducting research on the results of policies on Mental Health is debated. Increased research in this field could aid the region's health resource management.

\section{Keywords:}

Efectiveness in psychotherapy - Brief psychotherapy - Primary Health Care

Prof. Dr. Ignacio Barreira: https://orcid.org/0000-0002-2965-6412 ignacio.barreira@usal.edu.ar Licenciado, Profesor y Doctor en Psicología USAL. Docente e investigador por la USAL. Actualmente Director del Doctorado en Psicología USAL. Psicólogo clínico, planta en internación psiquiátrica, consulta particular. Actualmente Coordinador del Dispositivo de Rehabilitación de Sanatorio San Gabriel. Candidato en Posdoctorado en Psicología de UFLO. Recibido, 14/09/20, Publicado12/10/20 


\section{Introducción}

El siguiente estudio se basa en una revisión sistemática sobre evaluación en efectividad en psicoterapias breves en Iberoamérica. Se entiende por evaluación en efectividad en psicoterapias breves la ponderación objetiva del beneficio que estas prestaciones en salud mental generan en sus usuarios (Barreira, 2018). Los estudios sobre este tipo de tratamientos psicológicos se han orientado históricamente hacia la eficacia buscando establecer la validez interna de esos tratamientos, precisando los factores y las variables que forman parte de los mismos (Fernández-Álvarez y Castonguay, 2018). La prolífica generación de evidencia científica sobre la eficacia de estos tratamientos fue alimentando a los movimientos de medicina basada en la evidencia, impactando en la conformación de equipos que trabajan para establecer tratamientos psicológicos

validados. Por otra parte, este movimiento también fue visto con buenos ojos por parte de los administradores de recursos en Salud Mental, quienes empezaron a buscar garantías en los tratamientos que se debían cubri desde el estado o también desde empresas de medicina prepaga. Estos últimos, capitalizaron la producción referida en función de la relación costo-beneficio - eficiencia- de estos tratamientos (Polo-López et al., 2010). La eficacia debe entenderse en términos de

validez interna de los tratamientos-se ocupa de establecer si los tratamientos psicológicos realizan las acciones que se proponen y son replicables-, la efectividad se refiere a los efectos de los mismos-se enfoca en los resultados efectivos de los procedimientos implementados-, mientras que la eficiencia busca establecer la relación costo-beneficio en la implementación de las psicoterapias - pondera el beneficio efectivo de acuerdo con estas dos variables.

No obstante, los estudios de eficacia presentaron un impacto diferente en aquellos actores que se interesaban en el relevamiento de los resultados de los tratamientos psico-

dógicos-efectividad. Esto
ló resultados de los tratamientos psicoúltimo llevó a muchos clínicos e investigadores a la consideración de la a la considerán de la necesidad de genera evidencia de calidad sobre la efectividad de las psicoterapias breves al margen de los estudios de eficacia. Si bien los estudios en eficacia permitían estudiar los tratamientos psicológicos en diseños de investigación "de laboratorio", estos no garantizaban resultados efectivos ya que las consultas en diferentes contextos no necesariamente respetan las condiciones formales implementadas para investigar; os tratamientos psicológicos realizados de acuerdo a diversas realidades geopolíticas no ocurren en un laboratorio. En los último años el interés por evaluar la efectividad en los tratamientos psicológicos ha ido creciendo, desarrollándose, de acuerdo con corrientes específicas tales como las nucleadas en la noción de Treatment-As-Usual (TAU) (Löfholm et al., 2013), o el caso de la investigación orientada a la práctica, que intenta resolver la brecha entre pesquisa y práctica clínica efectiva (Fernández-Álvarez et al.,2020). Estas últimas tendencias buscan articular la práctica clínica tal como se realiza de hecho con la investigación rigurosa. De esta manera, se intenta preservar las condiciones espontáneas de trabajo entre paciente y terapeuta, evitando la invasión en los contextos clínicos en los que se realizan los tratamientos psicológicos, al tiempo que se posibilita la generación de evidencia de calidad sobre cuán efectivos estos resultan. En otro orden de cosas, estos mismos estudios deberían ser relevantes para establecer qué resultados se han obtenido como efecto de las políticas sanitarias implementadas en diferentes países de Iberoamérica, ya que los sistemas de Atención Primaria de la Salud cuentan generalmente con programas Salud Mental, y políticas específicas en relación con las psicoterapias breves tanto en el sector público como en el sector privado.

\section{Objetivo}

La presente revisión sistemática busca relevar las publicaciones sobre efectividad en psicoterapia breve en Iberoamérica.

\section{Método}

El presente estudio se realizó de acuerdo con los estándares de la declaración PRISMA (Urrutia y Bonfill, 2010; Munive-Rojas y Gutiérrez-Garibay, 2015), siguiendo los pasos de calidad para la revisión sistemática, con excepción de los específicos para estudios de revisión metaanalíticos, ítems $5,12,13,14,15,19,22$ y 27

\section{Procedimientos}

De acuerdo con el objetivo del presente estudio y en consonancia con el método utilizado de revisión sistemática, los ítems del método PRISMA aquí incluidos fueron: 1 (título), 2 (resumen estructurado), 3 (justificación), 4 (objetivo), 6 (criterios de elegibilidad), 7 (fuentes de información), 8 (búsqueda), 9 (selección de los estudios), 10 (proceso de extracción de datos), 11 (lista de datos), 16 (análisis adicionales) 17 (selección de estudios), 18 (características de los estudios), 20 (resultados de los estudios individuales), 21 (síntesis de los resultados), 23 (análisis adicionales), 24 (resumen de la evidencia), 25 (limitaciones), y 26 (conclusiones). Los excluyeron ocho ítems del declaración PRISMA específicos para revisiones de metaanálisis: 5 (protocolo y registro), 12 (riesgo de sesgo en los estudios individuales), 13 (medidas de resumen), 14 (síntesis de resultados), 15 y 22 (riesgo de sesgo entre los estudios) 19 (riesgo de sesgo en los estudios), 27 (financiación).

Se tuvieron en cuenta las siguientes bases de datos: Red Iberoamericana de Innovación y Conocimiento Científico (REDIB), Directory of Open Access Journal (DOAJ), Red de Revistas Científicas de América Latina y el Caribe (REDALYC), Scientific Electronic Library Online (ScIELO), DIALNET y el Portal Regional de la BVS de la Organización Panamericanal de la Salud. El criterio de selección de estas bases radicó en que estas reúnen una vasta cantidad de revistas que publican artículos altamente calificados para la tarea de revisión sistemática.

\section{Términos de búsqueda}

Con el objetivo de reclutar aquellas publicaciones que fueran pertinentes para la búsqueda, los términos considerados inicialmente para la misma fueron "efectividad en psicoterapia", "psicoterapia breve", "psicoterapia breve y focalizada", "efectividad en psicoterapia breve" y "efectividad en psicoterapia breve y focalizada". El motivo de elección de estos términos obedeció a que 
los mismos son los más adecuados para la búsqueda ensayada. La revisión sistemática de estas unidades de análisis se realizó en las bases REBID, DOAJ, REDALYC, ScIELO, DIALNET y Portal Regional en Salud de la Organización Panamericana de la Salud (OPS)

De acuerdo con diferentes búsqueda preliminares se trazó la estrategia para el estudio. Se desestimaron las unidades de análisis "psicoterapia breve y focalizada" "efectividad en psicoterapia breve y focalizada" por la escasez de resultados arrojados. Se consideró quitar de las búsquedas el término "focalizada" para lograr mejores resultados en las búsquedas. Consecuentemente, se partió de las unidades de análisis más genéricas ("efectividad en psicoterapia" y "psicoterapia breve"), estableciendo las búsquedas que relevaban mayor cantidad de estudios. Para el término "efectividad en psicoterapia" se relevaron 4276 resultados mientras que para "psicoterapia breve" se ubicaron 1787 .

A raíz de estos resultados se sintetizaron esas dos unidades de análisis en el término "efectividad en psicoterapia breve", que permitió mayor precisión en la búsqued arrojando 201 publicaciones. A estos resultados se aplicaron tres filtros para restringi la búsqueda de acuerdo con los objetivos del presente estudio: a) Tipo de publicación las unidades de análisis debían ser artículo científicos, excluyendo tesis, libros y capítulos de libros b) Lenguas de publicación espańol y portugués, excluyendo todas las otras lenguas. c) Período de publicación: últimos diez años, a partir de 2010 hasta el primer semestre de 2020. De este filtrado resultó una selección de 52 publicaciones, que aparecieron en REBID (5), DOAJ (1) REDALYC (5), ScIELO (3), DIALNET (19) y Portal Regional en Salud de la OPS (19). No hubo resultados en las bases REDALYCy SCIELO.
Al consultar los 52 estudios seleccionados, se identificaron cuatro duplicados, dos triplicados y dos cuadriplicados, debiéndose restar 14 unidades de análisis, dejando un remanente de 38 estudios. Los cuatro estudios duplicados pertenecieron a: dos trabajos replicados en DIALNET y Portal Regional en Salud de la OPS que se trataron de sendos estudios publicados en Actas españolas de psiquiatría (Lana Moliner et al., 2020), y Revista de Neurología (Serra Pla et al. 2017); un trabajo replicado en los buscadores REDIB y DIALNET publicado en la Revista Griot (Jurado Andino, 2017); y otro que fue referenciado por DIALNET en dos oportunidades, como habiendo sido publicado en dos revistas diferentes en los años 2016 y 2017, pero de manera errónea ya que se trataba del mismo trabajo que había sido publicado una única vez (Sánchez Arias, 2016).

Por su parte, los dos triplicados correspondieron a búsquedas en REDIB, DAO

y Portal Regional en Salud de la OPS, y ScIELO, DIALNET y Portal Regional en Salud de la OPS; se trató respectivamente de dos artículos pertenecientes a la Revista Electrónica Zoilo E. Marinello Vidaurreta (Limia Núnez et al., 2015), y a la Revista Salud Mental (Lara Muñoz et al., 2010). En el caso de los dos cuadriplicados se trató de dos trabajos ubicados por los buscadores REDIB, REDALYC, ScIELO y Portal Regional en Salud de la OPS, tratándose de producciones del mismo equipo, publicados sucesivamente en la Revista de la Asociación Española de Neurología (Fernández Méndez et al., 2010, 2011).

\section{Resultados}

El término de búsqueda "efectividad en psicoterapia breve", puede llegar a presenta resultados muy heterogéneos entre sí.

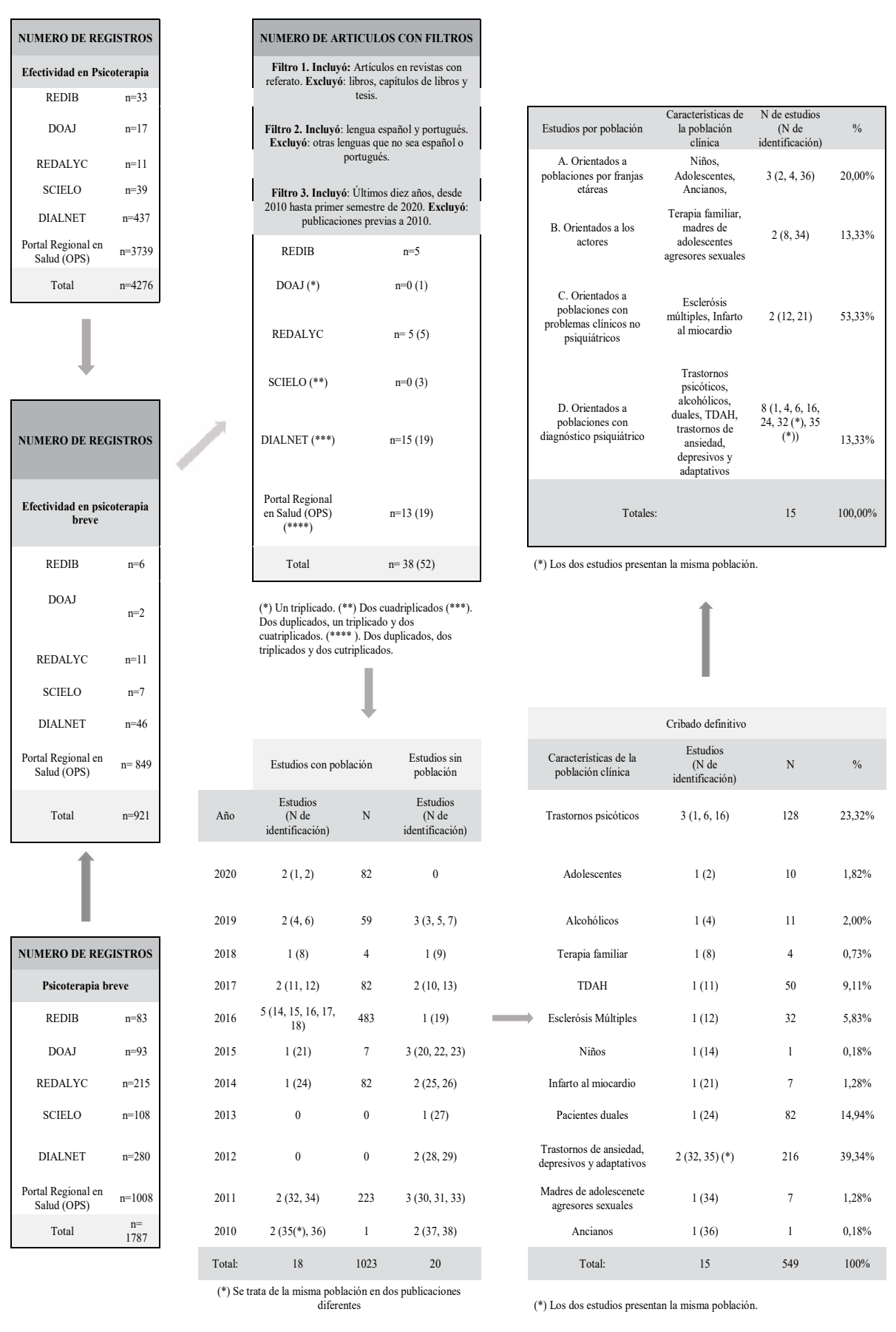

Revista Perspectivas Metodológicas | Universidad Nacional de Lanús | ISSN 2618-4125 
Una primera cuestión a resaltar consistió en advertir la distribución de las publicaciones por países para observar en qué lugar y proporción se dio lugar a este tipo de estudios. Los 38 estudios referidos fueron publicados en nueve países de Iberoamérica: 4 de Brasil, 3 de Colombia, 1 de Costa Rica, 3 de Cuba, 19 de España, 4 de México, 1 de Puerto Rico, 1 de Uruguay y 1 de EEUU. udio radicado en EEUU fue incluido por tratarse de una publicación de la Revista Panamericana de Salud Pública
(Rey et al., 2016). En esta distribución puede identificarse cómo el 50\% de la producción corresponde a publicaciones españolas (19 de las 38), 13,16\% a América del Norte (5 de 38), 13,16\% a América Central (5 de 38), y 23,68\% a América del Sur (9 de 38). Esto indica que no parece haber una gran producción de trabajos en lengua castellana en Iberoamérica, pero se advierte que en España hay mayor interés proporcional que en América Latina ya que, España iguala en cantidad la suma de todos los demás países.

\begin{tabular}{|c|c|c|c|c|c|c|c|c|}
\hline & $\begin{array}{c}\text { REDI } \\
\text { B }\end{array}$ & $\begin{array}{c}\text { DOA } \\
\mathrm{J}\end{array}$ & $\begin{array}{l}\text { REDALY } \\
\text { C }\end{array}$ & $\begin{array}{l}\text { ScIEL } \\
\mathrm{O}\end{array}$ & $\begin{array}{c}\text { DIALNE } \\
\mathrm{T} \\
\end{array}$ & $\begin{array}{c}\text { Portal } \\
\text { Regional } \\
\text { en Salud } \\
\text { (OPS) } \\
\end{array}$ & $\begin{array}{r}\begin{array}{c}\text { Subtotal } \\
\text { es por }\end{array} \\
\text { buscador }\end{array}$ & $\begin{array}{c}\text { Subtotal sin } \\
\text { duplicado ni } \\
\text { triplicado } \\
\end{array}$ \\
\hline Brasil & 0 & 0 & 2 & 0 & 1 & 1 & 4 & 4 \\
\hline Colombia & 0 & 0 & 0 & 0 & $4(*)$ & 0 & 4 & 3 \\
\hline Costa Rica & 0 & 0 & 1 & 0 & 0 & 0 & 1 & 1 \\
\hline Cuba & 2 & $1(*)$ & 1 & 0 & 0 & $2(* * *)$ & 6 & 4 \\
\hline España & 2 & 0 & 1 & $\begin{array}{c}2 \\
(* * *)\end{array}$ & $\begin{array}{l}10(*) \\
(* * * *)\end{array}$ & $11(* * * *)$ & 26 & 19 \\
\hline México & 0 & 0 & 0 & 1 & 3 & $3\left(^{* *}\right)$ & 7 & 4 \\
\hline Puerto Rico & 1 & 0 & 0 & 0 & $1\left(^{*}\right)$ & 0 & 2 & 1 \\
\hline Uruguay & 0 & 0 & 0 & 0 & 0 & 1 & 1 & 1 \\
\hline USA & 0 & 0 & 0 & 0 & 0 & 1 & 1 & 1 \\
\hline $\begin{array}{c}\text { Subtotales } \\
\text { por país }\end{array}$ & 5 & 1 & 5 & 3 & 19 & 19 & 52 & 38 \\
\hline $\begin{array}{c}\text { Subtotal sin } \\
\text { duplicado ni } \\
\text { triplicado }\end{array}$ & 5 & 0 & 5 & 1 & 15 & 12 & 38 & \\
\hline
\end{tabular}

Si se revisa la distribución de publicaciones por año de publicación, se puede advertir el siguiente detalle: 4 estudios de 2010, 5 estudios de 2011, 2 estudios de 2012, 1 estudio de 2013, 3 estudios de 2014, 4 estudios de 2015, 5 estudios de 2016, 5 estudios de 2017, 2 estudios de 2018, 5 estudios de

Revista Perspectivas Metodológicas | Universidad Nacional de Lanús | ISSN 2618-4125
2019 , y 2 estudios de 2020, Estos numeros, ton cantidad como en distribución, no permiten advertir una tendencia creciente o decreciente en los estudios.

\begin{tabular}{|c|c|c|c|c|c|c|c|c|c|c|c|c|c|}
\hline \multirow[b]{3}{*}{ Brasil } & & T & 3. I & Distri & ón & n de & studio & os por & ano $y$ & por $\mathrm{p}^{2}$ & & \multirow[b]{2}{*}{$\begin{array}{c}\text { Subtota } \\
\text { les por } \\
\text { país } \\
\end{array}$} & \multirow{2}{*}{$\begin{array}{l}\text { Subtota } \\
\text { sin } \\
\text { duplicad } \\
\text { o ni } \\
\text { triplicad } \\
\text { o } \\
\end{array}$} \\
\hline & $\begin{array}{c}201 \\
0\end{array}$ & $\begin{array}{c}201 \\
1\end{array}$ & $\begin{array}{c}201 \\
2\end{array}$ & $\begin{array}{c}201 \\
3\end{array}$ & $\begin{array}{c}201 \\
4\end{array}$ & $\begin{array}{c}201 \\
5\end{array}$ & $\begin{array}{c}201 \\
6\end{array}$ & $\begin{array}{c}201 \\
7\end{array}$ & $\begin{array}{c}201 \\
8\end{array}$ & $\begin{array}{c}201 \\
9\end{array}$ & $\begin{array}{c}202 \\
0\end{array}$ & & \\
\hline & 0 & 1 & 0 & 1 & 0 & 0 & 0 & 0 & 0 & 2 & 0 & 4 & 4 \\
\hline Colombia & 0 & 1 & 0 & 0 & 1 & 0 & 1 & $\begin{array}{c}1 \\
(*) \\
\end{array}$ & 0 & 0 & 0 & 4 & 3 \\
\hline Costa Rica & 0 & 0 & 0 & 0 & 1 & 0 & 0 & 0 & 0 & 0 & 0 & 1 & 1 \\
\hline Cuba & 1 & 0 & 0 & 0 & 0 & $\begin{array}{c}5 \\
(* * * \\
)\end{array}$ & 0 & 0 & 0 & 0 & 0 & 6 & 4 \\
\hline Espańa & $\begin{array}{c}5 \\
(* * * \\
*)\end{array}$ & $\begin{array}{c}5 \\
(* * * \\
*) \\
\end{array}$ & 2 & 0 & 1 & 0 & 2 & $\begin{array}{c}5 \\
\left(^{*}\right)\end{array}$ & 2 & 3 & 1 & 26 & 19 \\
\hline México & $\begin{array}{c}3 \\
(* *)\end{array}$ & 1 & 0 & 0 & 0 & 1 & 0 & 0 & 0 & 0 & $\begin{array}{c}2 \\
(*) \\
\end{array}$ & 7 & 4 \\
\hline Puerto Rico & 0 & 0 & 0 & 0 & 0 & 0 & 0 & $\begin{array}{c}2 \\
\left({ }^{*}\right)\end{array}$ & 0 & 0 & 0 & 2 & 1 \\
\hline Uruguay & 0 & 0 & 0 & 0 & 0 & 0 & 1 & 0 & 0 & 0 & 0 & 1 & 1 \\
\hline USA & 0 & 0 & 0 & 0 & 0 & 0 & 1 & 0 & 0 & 0 & 0 & 1 & 1 \\
\hline $\begin{array}{c}\text { Subtotales } \\
\text { por ańo }\end{array}$ & 9 & 8 & 2 & 1 & 3 & 6 & 5 & 8 & 2 & 5 & 3 & 52 & 38 \\
\hline $\begin{array}{l}\text { Subtotal sin } \\
\text { duplicado ni } \\
\text { triplicado }\end{array}$ & 4 & 5 & 2 & 1 & 3 & 4 & 5 & 5 & 2 & 5 & 2 & 38 & \\
\hline
\end{tabular}

De estos 38 estudios, se realizó un relevamiento de acuerdo a diferentes ejes que permitirían identificar especificidades de los mismos. En primer lugar, se establecieron las referencias formales de búsqueda: autores, año de publicación, título del estudio, país de publicación y buscador en que fue encontrado. Posteriormente, se establecieron los siguientes ejes para clasificar los estudios de acuerdo a sus contenidos: tipo de estudio / instrumentos, duración del estudio, modelo de intervención, tipo de intervención (dispositivo clínico), la población implicaday/o beneficiada y los resultados. Las variables

descriptas resultan altamente relevantes para poder calificar el tipo de estudios, y genera una idea consistente en relación al tipo de estudios que se realizaron en Iberoamérica en los últimos 10 años. 
Tabla 4. Deralle de essudios por Autror, Año, Tírulo, Tipo de Essudio, Poblacion y Resultados

\begin{tabular}{llcll} 
Autror / año & Tírulo & $\begin{array}{c}\text { Tipo de Essudio I } \\
\text { Instrumentros }\end{array}$ & Población & Recultados/Condusiones \\
\hline
\end{tabular}

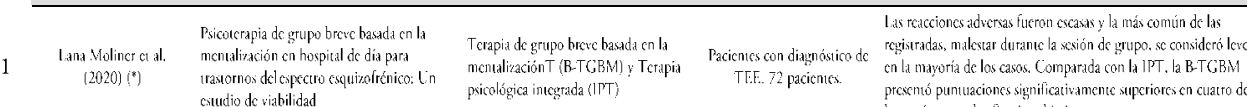

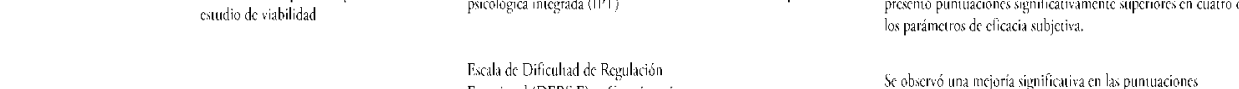

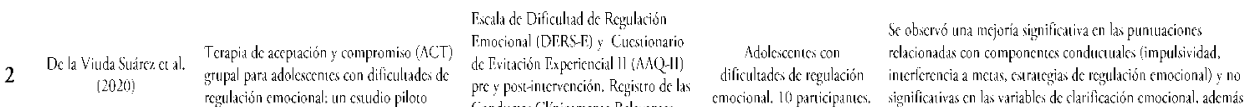

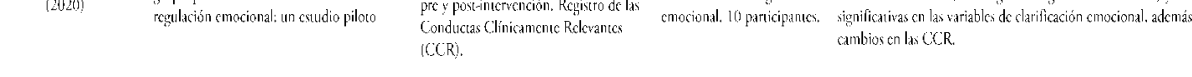

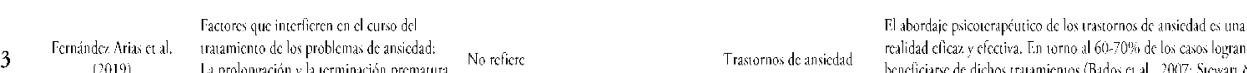

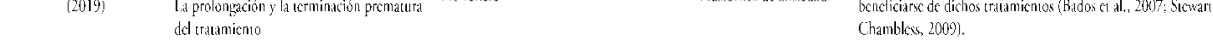

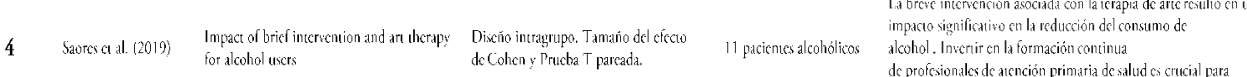

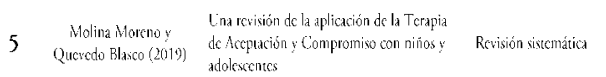

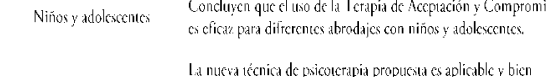

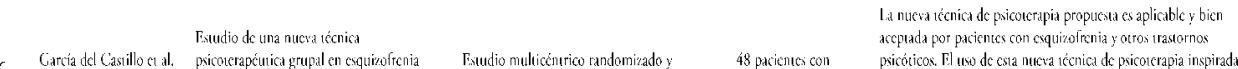

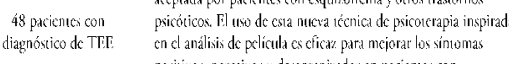

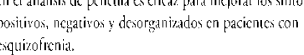

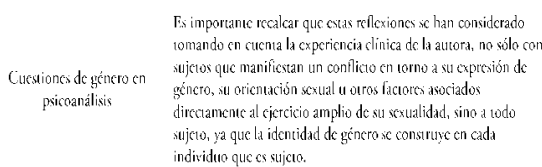

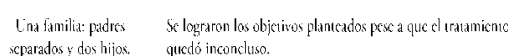

Sin preciart Sc restala laing

(2al18)

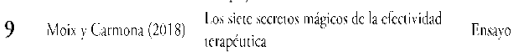

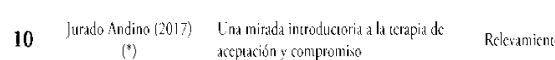

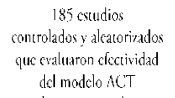

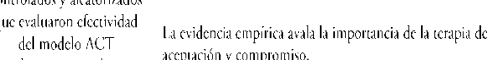

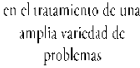

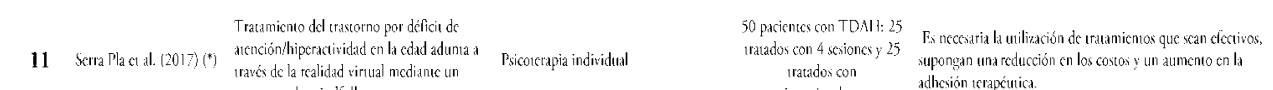

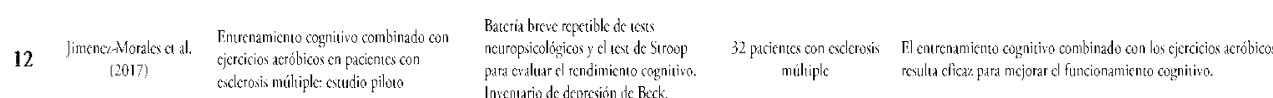

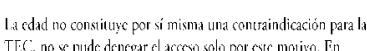

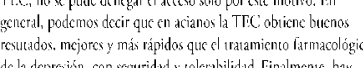

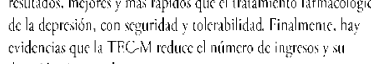

13 Alexerer Iryuty

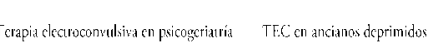

Ancianos deppinimid

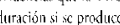

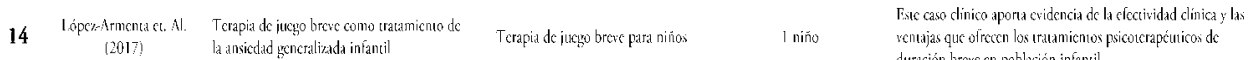

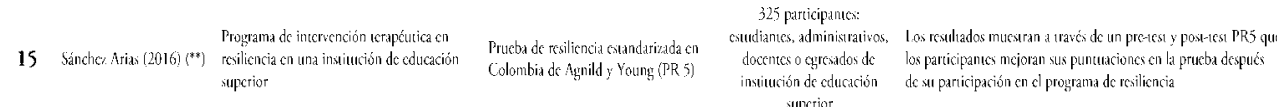

Revista Perspectivas Metodológicas | Universidad Nacional de Lanús | ISSN 2618-4125

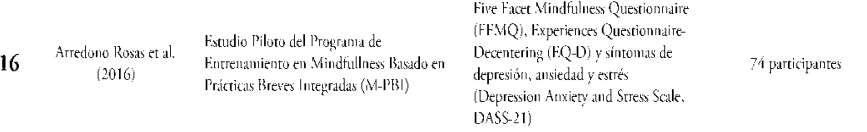

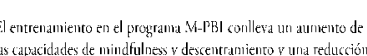

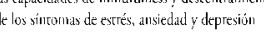

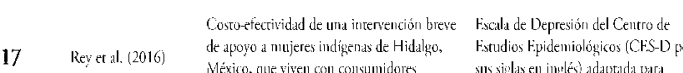

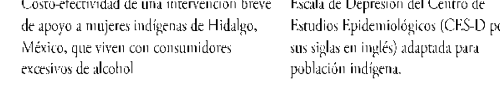

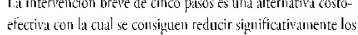

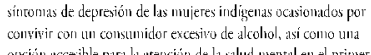

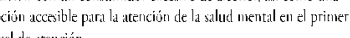

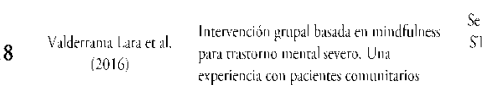

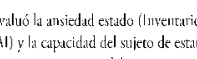

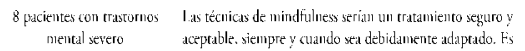

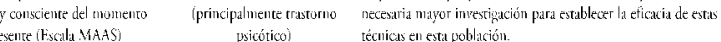

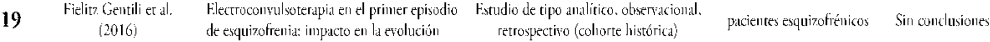

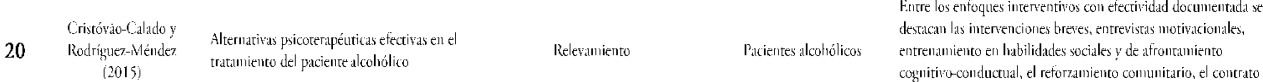

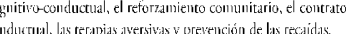

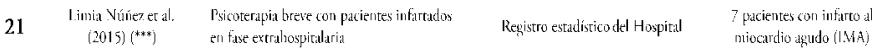

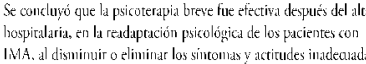

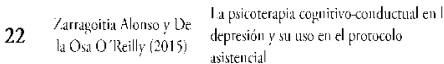

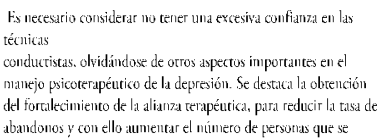

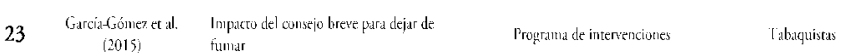

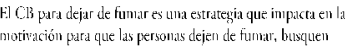

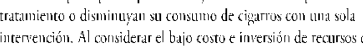

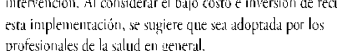

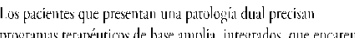

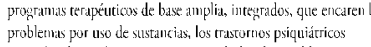

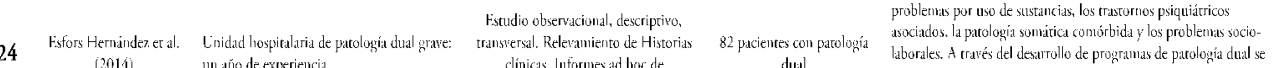

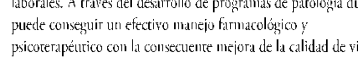

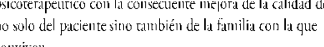

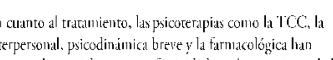

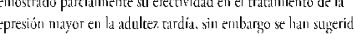

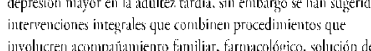

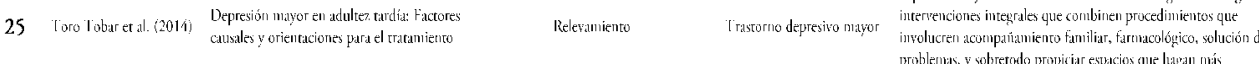

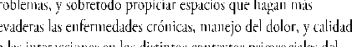

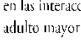

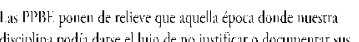

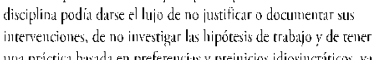

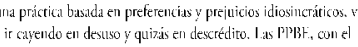

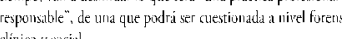

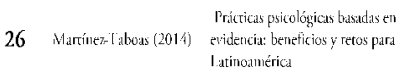

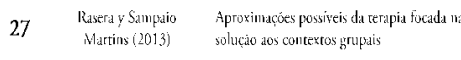

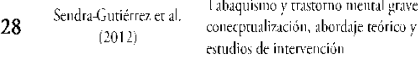

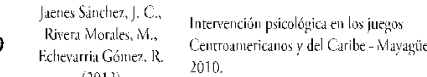

Revisión

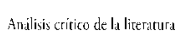

Revista Perspectivas Metodológicas | Universidad Nacional de Lanús | ISSN 2618-4125 


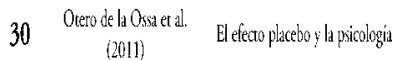

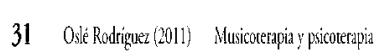

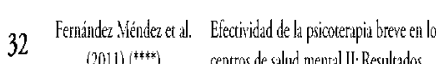

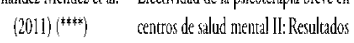

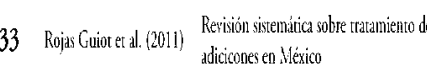

Parricipacio de finilias no Grupo

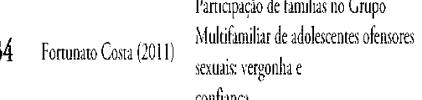

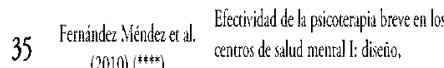

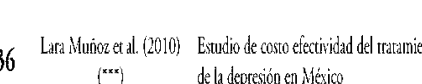

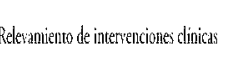

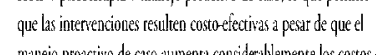

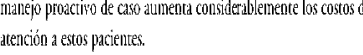

Estudio descripirivo, culliatirio. El

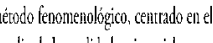

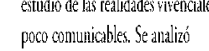

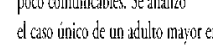
exraxodef fingithdid.

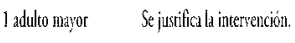

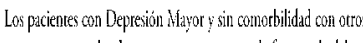

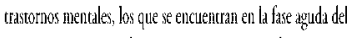

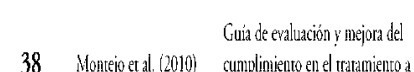

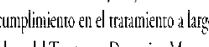
Rerision

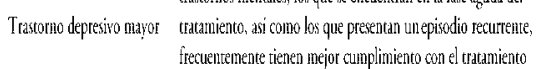
anididererivio.

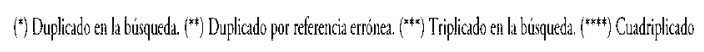

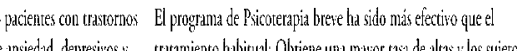

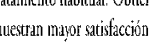

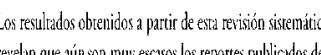

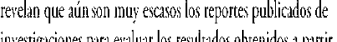

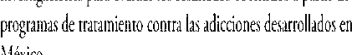

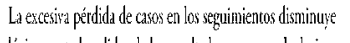

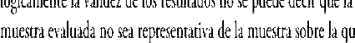
pretendens sacr ondlusiones.

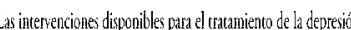

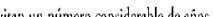

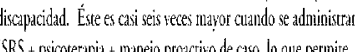

Como consecuencia de ese análisis, se detectó que varios estudios ubicados no presentaban población clínica; es decir, se trataba de estudios que no contaban con participantes y/o pacientes. Veinte de estos estudios eran revisiones, relevamientos, análisis críticos de literatura o reportes. Se trataba de trabajos que presentaban generalidades sobre aspectos relevantes de la psicoterapia, por su cualidad metodológica en relación con un enfoque teórico o una modalidad, o por su importancia en relación con ciertas poblaciones de pacientes.

\begin{tabular}{|c|c|c|c|}
\hline \multicolumn{4}{|c|}{ Tabla 5. Estudios con o sin población } \\
\hline & \multicolumn{2}{|c|}{ Estudios con población } & \multirow{2}{*}{$\begin{array}{l}\text { Estudios sin } \\
\text { población } \\
\mathrm{N} \text { de estudios } \\
\text { ( } \mathrm{N} \text { de } \\
\text { identificación) }\end{array}$} \\
\hline Año & $\begin{array}{l}\mathrm{N} \text { de estudios } \\
\text { ( } \mathrm{N} \text { de } \\
\text { identificación) }\end{array}$ & $\begin{array}{c}\mathrm{N} \text { de } \\
\text { participantes }\end{array}$ & \\
\hline 2020 & $2(1,2)$ & 82 & 0 \\
\hline 2019 & $2(4,6)$ & 59 & $3(3,5,7)$ \\
\hline 2018 & $1(8)$ & 4 & $1(9)$ \\
\hline 2017 & $2(11,12)$ & 82 & $2(10,13)$ \\
\hline 2016 & $\begin{array}{c}5(14,15,16 \\
17,18)\end{array}$ & 483 & $1(19)$ \\
\hline 2015 & $1(21)$ & 7 & $3(20,22,23)$ \\
\hline 2014 & $1(24)$ & 82 & $2(25,26)$ \\
\hline 2013 & 0 & 0 & $1(27)$ \\
\hline 2012 & 0 & 0 & $2(28,29)$ \\
\hline 2011 & $2\left(32\left(^{*}\right), 34\right)$ & 223 & $3(30,31,33)$ \\
\hline 2010 & $2\left(35\left(^{*}\right), 36\right)$ & 1 & $2(37,38)$ \\
\hline Total: & 18 & 1023 & 20 \\
\hline
\end{tabular}

$\left(^{*}\right)$ Se trata de la misma población en dos publicaciones diferentes
Dentro de los estudios que se centraban en las cualidades metodológicas de las psicoterapias se pudieron ubicar aquellos que resaltaban la importancia de las prácticas psicológicas basadas en la evidencia (Martínez-Taboas, 2014), los factores inespecíficos en psicoterapia desde una perspectiva jungiana (Moix y Carmona, 2018), un modelo de psicoterapia basada en soluciones (Rasera y Sampaio Martins, 2013), la importancia de considerar las cuestiones de género en psicoanálisis (Garay, 2019), la relación entre la psicoterapia y la musicoterapia (Oslé Rodríguez, 2011), y la necesidad en investigar el efecto placebo y la psicoterapia (Otero de la Ossa et al., 2011). En relación a los estudios que se basaron en la consideración de las psicoterapias en relación con cuadros psicopatológicos, se ubicaron trabajos sobre tabaquismo (Sendra-Gutiérrez et al., 2012, García-Gómez et al., 2015), alcoholismo (Cristóváo-Caado y Rodríguez-Méndez, 2015), abuso de sustancias en general (Rojas Guiot et al., 2011) esquizofrenia (Fielitz Gentili et al., 2016), depresión (Montejo et al., 2010, Lara Mu- 
ñoz et al., 2010, Toro Tobar et al., 2014), Zarragoitia Alonso y De la Osa O'Reilly, 2015), o trastornos de ansiedad (Fernández Arias et al., 2019). Por último, dos estudios hicieron mención respectivamente a psicoterapias orientadas al trabajo con nińos adolescentes (Molina Moreno y Quevedo Blasco, 2019), y orientadas al trabajo con ancianos (Alcoverro Fortuny, 2017). Esto 20 estudios resultan relevantes en diferentes sentidos, pero no constituyen relevancia de acuerdo con objetivo del estudio, debido a que los mismos son formulaciones genéricas sobre la importancia de diferente aspectos de métodos psicoterapéuticos, pero no constituyen estudios formales sobre efectividad en psicoterapia breve. De hecho, la caracterización general de esto estudios radica más en cómo rescatar ciertas dimensiones o modos de considerar diversos aspectos de métodos psicoterapéuticos, pero ninguno de ellos establece un diseño en el que se aplican tratamientos y se evalúa la efectividad de los mismos.

Los 18 estudios restantes presentaron investigaciones con participantes, aunque no todos los estudios encontrados eran de psicoterapias. Tres de estos 18 estudios consistían en investigaciones sobre poblaciones de personas que participaron en calidad de concurrentes, se trató de 325 personas que participaron en un programa de intervención psicoterapéutica en resiliencia en una institución de educación superior, en donde la población estaba constituida por estudiantes, administrativos, docentes y egresados (Sánchez Arias, 2016), 74 participantes en un estudio piloto en un programa de entrenamiento en mindfullness basado en prácticas breves integradas (Arredono Rosas et al., 2016) y 73 participantes de un estudio social que eran mujeres indígenas que convivían con consumidores excesivos de alcohol (Rey et al., 2016). Estos tres estudios se salen de la línea de trabajos de investigación en dispositivos de psicoterapia habituales en

Revista Perspectivas Metodológicas | Universidad Nacional de Lanús | ISSN 2618-4125 los que se lo investigado está centrado en la efectividad de las psicoterapias.

Los 15 trabajos restantes presentaron estudios con diferentes poblaciones de pacientes. En principio podemos establecer tres grandes grupos: a) orientado rias, b) orientados a poblaciones en lo que se destaca el rol de los participantes, c) orientados a poblaciones con problemas clínicos no psiquiátricos y, d) orientados a poblaciones con diagnóstico psiquiátrico. a poblaciones definidas por franjas eta-

Tabla 6. Clasificación de los estudios del cribado definitivo

\begin{tabular}{|c|c|c|c|}
\hline $\begin{array}{l}\text { Estudios por } \\
\text { población }\end{array}$ & $\begin{array}{c}\text { Características de la } \\
\text { población }\end{array}$ & $\begin{array}{l}\mathrm{N} \text { de estudios } \\
\text { ( } \mathrm{N} \text { de } \\
\text { identificación) }\end{array}$ & $\%$ \\
\hline $\begin{array}{l}\text { A. Orientados a } \\
\text { poblaciones por } \\
\text { franjas etarias }\end{array}$ & $\begin{array}{c}\text { Niños, Adolescentes, } \\
\text { Ancianos, }\end{array}$ & $3(2,4,36)$ & $20,00 \%$ \\
\hline $\begin{array}{l}\text { B. Orientados a los } \\
\text { participantes }\end{array}$ & $\begin{array}{c}\text { Terapia familiar, madres } \\
\text { de adolescentes agresores } \\
\text { sexuales }\end{array}$ & $2(8,34)$ & $13,33 \%$ \\
\hline $\begin{array}{c}\text { C. Orientados a } \\
\text { poblaciones con } \\
\text { problemas clínicos no } \\
\text { psiquiátricos }\end{array}$ & $\begin{array}{l}\text { Esclerósis múltiples, } \\
\text { Infarto al miocardio }\end{array}$ & $2(12,21)$ & $13,33 \%$ \\
\hline \multirow[t]{2}{*}{$\begin{array}{l}\text { D. Orientados a } \\
\text { poblaciones con } \\
\text { diagnóstico } \\
\text { psiquiátrico }\end{array}$} & $\begin{array}{l}\text { Trastornos psicóticos, } \\
\text { alcohólicos, duales, } \\
\text { TDAH, trastornos de } \\
\text { ansiedad, depresivos y } \\
\text { adaptativos }\end{array}$ & $\begin{array}{l}8(1,4,6,16 \\
24,32\left(^{*}\right), 35 \\
\left.\left(^{*}\right)\right)\end{array}$ & $53,33 \%$ \\
\hline & Totales: & 15 & $100,00 \%$ \\
\hline
\end{tabular}

$\left(^{*}\right)$ Los dos estudios presentan la misma población. a) De los estudios orientados a poblaciones definidas por franjas etarias se pueden ubicar tres: un estudio sobre niños (López-Armenta et. al., 2017), uno con adolescentes (De la Viuda Suárez et al., 2020), y uno con ancianos (Lara Muñoz et al., 2010). Estos estudios intentaron identificar aspectos o peculiaridades que fueran propios de desarrollo, de modo tal que se pudieran ayudar en una eventualidad cualificación aspectos comunes de estas poblaciones. b) De aquellos que estuvieron orientados a poblaciones en los que se destacaba el rol de los participantes, se identificaron dos: un estudio de terapia familiar que se trataba de una única familia compuesta por padre, madre y dos hijos (Castillo Sotelo et al., 2018), y un estudio de madres de agresores sexuales (Fortunato Costa, 2011).
En ambos estudios se destacó el modo en que se definió a las unidades de análisis de manera diferente entre sí, pero recayendo en un grupo y no en una persona, pese a que se trató de un caso de una única familia, y madres de agresores, de modo tal que el foco de la investigación se orientaba a identificar peculiaridades de estas madres que pudieran establecer eventualmente algún patrón o regularidad en estas que, eventualmente pudieran vincularse con la agresividad sexual de sus hijos. c) los estudios orientados a poblaciones con problemas clínicos no psiquiátricos fueron dos: un caso de esclerosis múltiple (Jimenez-Morales et al., 2017), y un estudio con infartados (Limia Núñez et al., 2015). Estos estudios se orientaron a establecer si era posible identifica perfiles psicológicos en este tipo de pacientes clínicos. Por último, d) Los trabajos orientados 
a poblaciones con diagnóstico psiquiátrico fueron los más cuantiosos, se ubicaron ocho en total: tres estudios presentaron casos de esquizofrenia (Arredono Rosas et al., 2016 García del Castillo et al., 2019, Lana Moliner et al. 2020), un estudio sobre pacientes alcohólicos (Saores et al., 2019), uno con pacientes duales (Toro Tobar et al., 2014), uno sobre trastorno de déficit atencional con hiperactividad (Serra Pla et al., 2017), y dos estudios con una población de pacientes con diagnósticos de ansiedad, depresión y trastornos adaptativos (Fernández Méndez et al., 2010, 2011). De estos ocho estudios, el último fue en realidad un mismo grupo de investigación que presentó en dos trabajos la planificación en 2010 y los resultados en 2011. Consecuentemente, quedaron 7 estudios en los que se destacan tres estudios con pacientes psicóticos y uno con pacientes duales. Estos trabajos representan lo que habitualmente se entiende en sentido estricto como estudios de efectividad en psicoterapia breve y focalizada ya que cuentan con poblaciones de pacientes con diagnóstico psicopatológico, se aplicaron tratamientos en salud mental y se evaluaron los resultados de los mismos. Cabe resaltar que estas ocho publicaciones correspondieron seis a revistas españolas, una publicación mexicana y una publicación brasilera.

\section{Discusión}

La presente revisión sistemática sobre evaluación de efectividad en psicoterapia breves y focalizadas resulta importante a fines de relevar las publicaciones que generan evidencia de calidad sobre el beneficio que este tipo de tratamientos proveen a diferentes poblaciones en Iberoamérica. A diferencia de los estudios de eficacia que ponen el foco en la arquitectura interna de este tipo de tratamientos, los estudios de efectividad intentan establecer cuál es el resultado concreto de los tratamientos que los usuarios en salud mental realizan, cuestión que va mucho más allá de discusiones sobre el diseño de los mismos. La importancia de estos estudios radica en generar evidencia de calidad sobre el beneficio que estas prestaciones en salud mental aportan a la población en general por lo que resulta muy significativo que las políticas sanitarias que propongan este tipo de prestaciones realicen estos estudios para sacar en limpio el impacto concreto de estos tratamientos en la población. De este modo, relevar las publicaciones científicas sobre este tema en países que implementan este tipo de políticas en Atención Primaria de la Salud, se torna relevante a fines de establece qué tipo de evaluaciones se realizan sobre las medidas implementadas para beneficio de las diferentes poblaciones.

De acuerdo con los resultados presentados cabe destacar los pocos estudios hallados en relación con la investigación de efectividad en las psicoterapias breves en Iberoamérica. Resulta llamativo que estos dispositivos en salud mental presenten pocas publicacione cuando las políticas en Atención Primaria de Salud en estas regiones disponen de planes proactivos en la implementación de este tipo de prestaciones para la población en general. Si consideramos que partimos de una búsqueda de estudios sobre "efectividad en psicoterapia breve" que arrojó 921 unidades de análisis, el hecho de que hubieran quedado solo ocho estudios correspondientes a siet equipos de investigación, que fueran los que dan cuenta precisamente de este fenómeno, resulta cuanto menos escaso de acuerdo con las políticas sanitarias iberoamericanas. $\mathrm{Si}$ además tenemos en cuenta que estas ocho publicaciones corresponden seis a Europa, una a América Central y una a América del Sur, con la peculiaridad de que el último estudio es brasilero-que no fue publicado en lengua castellana, resulta muy sorprendente la nula tendencia en relación a la realización y/o publicación de este tipo de estudios. Sobre todo teniendo en cuenta que los propios sistemas de Atención Primaria de Salud deberían estar muy interesados en evaluar los resultados de las políticas que se implementan. El problema es que al no testearse la efectividad de estas políticas, se corre el riesgo de llevar adelante políticas ciegas.

Las poblaciones de los estudios que quedaron del cribado final correspondieron tres estudios a pacientes psicóticos, un estudio a pacientes duales, un estudio en pacientes alcohólicos, un estudio de trastornos por déficit atencional con hiperactividad y dos estudios con pacientes con trastornos depresivos, por ansiedad y adaptativos. Si lo que está en juego en este tipo de tratamientos es su importancia en la democratización de la salud mental, beneficiar a la mayor cantidad de personas posibles en una comunidad de un modo equitativo (Barreira, 2018), resulta relevante que la efectividad sea evaluada en diferentes poblaciones de personas con diversos padecimientos psicopatológicos. En este sentido, se considera altamente significativo que se estudien psicoterapias breves en cuadros psicopatológicos con gravedad severa y moderada como lo constituyen los casos de pacientes psicóticos o duales, porque es el modo en poder identificar si estos abordajes tienen sentido, o deben articularse en otro tipo de abordajes orientados a la prevención sanitaria secundaria o terciaria. Esto también sucede con pacientes alcoholistas, que muchas veces son diagnosticados por alcoholismo, pero también como trastornos de la personalidad. En relación con los estudios sobre pacientes con trastornos depresivos, trastornos de ansiedad y trastornos adaptativos, resulta quizás la población más adecuada para evaluar efectividad debido a que estos trastornos mentales leves a moderados, son los más adecuados para ser absorbidos en dispositivos de Atención Primaria de Salud, y son los que a priori, deberían tener mayor éxito. Las intervenciones de psicoterapias breves y focalizadas han sido ideadas originalmente con el objetivo de dar respuesta rápida a la generalidad de la población, consecuentemente, aquellos cuadros psicopatológicos que presenten una gravedad menor, serán más adecuados para ser tratados efectivamente en menor tiempo. Es por eso que los estudios de Fernández Méndez et al. (2010,2011), resultan altamente relevantes y no es ingenuo que los mismos hubieran aparecido por cuadruplicado en los buscadores REDIB, REDALYC, DIALNET y Portal Regional en Salud (OPS). Esto mismo vale para el estudio deTDAH de Serra Plat et al. (2017), que fue ubicado duplicado en DIALNET y Portal Regional en Salud (OPS).

Las limitaciones del presente estudio recaen en la falta de búsqueda en trabajos en otras lenguas que no fueran el castellano y el portugués. Esto implica que pueden existir equipos de investigación iberoamericanos que eventualmente pudieran haber realizado publicaciones en otras lenguas, aunque estratégicamente resultaría extraño, debido a que resultados sobre efectividad en psicoterapias de países de Iberoamérica implicaría que los mismos pudieran circular en sus comunidades siendo publicadas en sus lenguas vernáculas. No obstante, esta limitación incide en el hecho de que podrían buscarse y encontrarse modelos alternativos a la evaluación de efectividad en psicoterapias breves.

\section{Bibliografía}

— Alcoverro Fortuny, O. (2017). Terapia electroconvulsiva en psicogeriatría. Inf. Psiquiátr., 228, 77-86.

— Arredondo Rosas, M., Sabaté Pina, M. Botella García del Cid, L, Acosta, L. M. y Hurtado, P. (2016). Estudio Piloto del Programa de Entrenamiento en Mindfullness Basado en Prácticas Breves Integradas 
(M-PBI). Revista de Psicoterapia, 27 (103), 151-168.

Barkham, M., Delgadillo, J., Firth, N., y Saxon, D. (2018). La evidencia basada en la práctica y la ley de variabilidad en el tratamiento psicológico. Revista Argentina de Clínica Psicológica, XXVII (2), 115-135.

— Barreira, I. (2018). Terapias breves y focalizadas en Argentina. Enciclopedia Argentina en Salud Mental.

— Castillo Sotelo, M., Brito Sánchez, A. I., y Vargas Jiménez, R. (2018). Enfoque breve estratégico: un camino simple y efectivo para solucionar problemas complejos. Revista de psicoterapia, 29 (111), 257-271.

—Cristóvão-Calado, J. M., y RodríguezMéndez, C. L. (2015). Alternativas psicoterapéuticas efectivas en el tratamiento del paciente alcohólico. Gaceta Médica Espirituana, 17 (2).

— De la Viuda Suárez, M. E., y Casas Posada, A. (2020). Terapia de aceptación y compromiso (ACT) grupal para adolescentes con dificultades de regulación emocional: un estudio piloto. Revista de Psicología Clínica con Niñosy Adolescentes, 7 (2), 42-49.

— Esfors Hernández, J., Villar García, M. Rubio Perlado, B., Juan i Porcar, M., y Romero Marmaneu, F. (2014). Unidad hospitalaria de patología dual grave: un ańo de experiencia. Revista española de drogadependencias, 1, 31-44.

— Fernández-Álvarez. H., y Castonguay, L. G. (2018). Investigación orientada por la práctica: avances en colaboraciones entre clínicos e investigadores. Revista Argentina de clínica psicológica, XXVII (2), 1007-14.

— Fernández-Álvarez, H., Prado-Abril, J., Sánchez-Reales, S., Molinari, G. Gómez Penedo, J. M., y Jeong Youn, S. (2020). La brecha entre la investigación y la práctica clínica: hacia la integración de la psicoterapia. Pap. Psicol. En prensa.

Revista Perspectivas Metodológicas | Universidad Nacional de Lanús | ISSN 2618-4125
- García-Gómez, L., Sansores, R. H., Noé Díaz, V., Urdapilleta-Herrera, E., González-Rojas, G., Pérez-Márquez, L. E., y Ramírez Venegas, A. (2015). Impacto del consejo breve para dejar de fumar. Salud Pública Mex., 57 (5), 366-7.

— Jaenes Sánchez, J. C., Rivera Morales, M., y Echevarria Gómez, R. (2012). Intervención psicológica en los juegos Centroamericanos y del Caribe - Mayagüez 2010. Revista de psicología del deporte, 21 (1), 177-181.

- Jiménez Betancourt, E., e Írsula Betancourt, L. (2010). Adulto mayor en estado de fragilidad. MEDISAN, 14 (3), 396-401.

- Jimenez-Morales, R. M., HerreraJimenez, L. F., Macias-Delgado, Y., Perez-Medinilla, Y.T., Diaz-Diaz, S. M., y Forn, C. (2017). Entrenamiento cognitivo combinado con ejercicios aeróbicos en pacientes con esclerosis múltiple: estudio piloto. Rev. neurol., 64 (11), 489-495.

— Jurado Andino, M. (2017). Una mirada introductoria a la terapia de aceptación y compromiso. Griot, 10 (1), 70-87.

- Lara Muñoz, M. C., Robles García, R., Orozco, R., Real Fortuny, T., Chisholm, D., y Medina-Mora Icaza, M. E. (2010). Estudio de costo efectividad del tratamiento de la depresión en México. Salud Mental, 33 (4), 301-308.

_- Lana Moliner, F., Martí Bonany, J., Sanz Correcher, P, Pérez Solá, V., y Irimia, A. (2020). Psicoterapia de grupo breve basada en la mentalización en hospital de día para trastornos del espectro esquizofrénico: Un estudio de viabilidad. Actas españolas de psiquiatría, 48, (2), 64-74.

- Limia Núñez, Y.; Vargas González, D., y

Núñez Quintero, M. (2013). Psicoterapia breve con pacientes infartados en fase extrahospitalaria. Revista Electrónica Dr. Zoilo E. Marinello Vidaurreta, 38 (4).
— Löfholm, C. A., Brännström, L., Olsson, M. \& Hansson, K. (2013). Treatment-asusual in effectiveness studies: What is it and does it matter? International Journal of Social Warefare, 22 (1), 25-34

— López-Armenta, F., Guerrero Guerra, D., y Gómez Cotero, A. (2017). Terapia de juego breve como tratamiento de la ansiedad generalizada infantil. Rev. Psicopatol. Salud ment. Niño adolesc., (29), 37-46.

- Martínez-Taboas, A. (2014). Prácticas psicológicas basadas en la evidencia: beneficios y retos para Latinoamérica. Revista Costarricense de Psicología, 33 (2), 63-78.

— Moix, J., y Carmona, V. (2018). Los siete secretos mágicos de la efectividad terapéutica. Papeles del Psicólogo, 39 (1).

- Molina Moreno, P., y Quevedo Blasco, R. (2019). Una revisión de la aplicación de la Terapia de Aceptación y Compromiso con niños y adolescentes. Int. j. psychol. psychol. ther., 19 (2), 173-188.

— Montejo, Á. L; Menchón, J. M.; Carrasco, J. L; Franco, M.; Martín Carrasco, M., y Moriñigo, A. (2010). Guía de evaluación y mejora del cumplimiento en el tratamiento a largo plazo del Trastorno Depresivo Mayor. Actas Esp. Psiquiatr., 38 (Supl. 2), 1-27.

-Munive-Rojas, S., y Gutiérrez-Garibay, M. (2015). ¿Cómo realizar una revisión sistemática y meta-análisis? Basado en la declaración PRISMA (Preferred Reporting Items for Systematic reviews and Meta-Analyses), directrices para la publicación de revisiones sistemáticas y meta-análisis de estudios que evaluan intervenciones sanitarias. Rev Card CM Ins Nac Card, 2, 32-37.

- Oslé Rodríguez, R. (2011). Musicoterapia y psicoterapia. Avances en salud mental relacional, 10 (2).

- Otero de la Ossa, Y. M, Munive Escorcia, J. C., y Ayala Sánchez, E. A. (2011). E efecto placebo y la psicología. Duazary: Revist 
internacional de Ciencia de la Salud, 8 (1), 112-114.

- Rasera, E. F., y Sampaio Martins, P. P. (2013). Aproximaçôes possíveis da terapia focada na solução aos contextos grupais. Psicologia: Ciência e Profissão, 33 (2), 318-335.

Rey, G. N., Aguilar, P. S., Pérez, F. C., Orford, J., Escudero, G., y Sainz, M. T. (2016).Costo-efectividad de una intervención breve de apoyo a mujeres indígenas de Hidalgo, México, que viven con consumidores excesivos de alcohol. Rev Panam Salud Publica, 39 (2), 69-75.

Rojas Guiot, E., Real Fortuny, T., García Silberman, S., y Medina-Mora Icaza, M. E. (2011). Revisión sistemática sobre tratamiento de adicciones en México. Salud Mental, 34 (4), 351-365.

—- Sánchez Arias, G. (2016). Programa de intervención terapéutica en resiliencia en una institución de educación superior. Revista de Psicología, 8 (1), 49-64.

— Serra Pla, J. F., Pozuelo, M, Richarte, V. Corrales de la Cruz, M., Ibáñez, P, Bellina, M., Vidal, R., Calvo, E., Casas Brugué, M., y Ramos-Quiroga, J.A. (2017). Tratamiento del trastorno por déficit de atención/hiperactividad en la edad adulta a través de la realidad virtual mediante un programa de mindfullness. Revista de neurologia, 64 (Extra 1), 117-122.

- Sendra-Gutiérrez, J. M, Casanova Peña, B., y Vargas Aragón, M. L. (2012). Tabaquismo y trastorno mental grave: conecptualización, abordaje teórico y estudios de intervención. Revista de la Asociación Española de Neuropsiquiatría, 32 (116), 707-722.

Soares, M. H., Rolin, T. F., Machado, F. P., Ramos, L. K., y Rampazzo, A. R. (2019). Impact of brief intervention and art therapy for alcohol users. Rev. Bras. Enferm., 72 (6), 1485-1489.
— Toro Tobar, R., Vargas, J. Murcia, S., Gaitán, N., Umbarila, D., y Sarmiento, K. (2013). Depresión mayor en adultez tardía: Factores causales y orientaciones para el tratamiento. Revista Vanguardia Psicológica Clínica Teórica y Práctica, 4 (2), 147-153.

— Urrutia, G., y Bonfill, X. (2010). Declaración PRISMA: una propuesta para mejorar la publicación de revisiones sistemáticas y metaanálisis. Med Clin, 135 (11), 507-511.

- Valderrama Lara, K., Rodriguez Castañeda, M. M., y Gonzalez Suarez, B. (2016). Intervención grupal basada en mindfulness para trastorno mental severo. Una experiencia con pacientes comunitarios. Inf. Psiquiátr, (225), 47-57.

Zarragoitia Alonso, I., y De la Osa O’Reilly, M. (2015). La psicoterapia cognitivo-conductual en la depresión y su uso en el protocolo asistencial. Análisis crítico. Rev. Hosp. Psiquiátr., 12 (2). 